\title{
Potential effectiveness and safety of antiviral agents in children with coronavirus disease 2019: a rapid review and meta-analysis
}

\author{
Qianling Shi' ${ }^{1 \#}$, Qi Zhou ${ }^{1,2 \#}$, Xia Wang ${ }^{3,4,5 \#}$, Jing Liao ${ }^{3,4,5}$, Yang Yu ${ }^{6}$, Zijun Wang ${ }^{2}$, Shuya Lu $^{7,8}$, \\ Yanfang $\mathrm{Ma}^{2}$, Yangqin Xun ${ }^{2}$, Xufei Luo,9, Weiguo $\mathrm{Li}^{3,4,5}$, Toshio Fukuoka ${ }^{10,11}$, Hyeong Sik Ahn ${ }^{12,13}$, \\ Myeong Soo Lee ${ }^{14,15}$, Zhengxiu Luo ${ }^{3,4,5}$, Enmei Liu ${ }^{3,4,5}$, Yaolong Chen ${ }^{2,16,17,18}$, Qubei Li ${ }^{3,4,5}$, Kehu Yang ${ }^{1,2,18}$, \\ Quanlin Guan ${ }^{1,19}$; on behalf of COVID-19 Evidence and Recommendations Working Group
}

\begin{abstract}
${ }^{1}$ The First School of Clinical Medicine, Lanzhou University, Lanzhou 730000, China; ${ }^{2}$ Evidence-based Medicine Center, School of Basic Medical Sciences, Lanzhou University, Lanzhou 730000, China; ${ }^{3}$ Department of Respiratory Medicine, Children's Hospital of Chongqing Medical University, Chongqing 400014, China; ${ }^{4}$ National Clinical Research Center for Child Health and Disorders, Ministry of Education Key Laboratory of Child Development and Disorders, China International Science and Technology Cooperation Base of Child Development and Critical Disorders, Children's Hospital of Chongqing Medical University, Chongqing 400014, China; 'Chongqing Key Laboratory of Pediatrics, Chongqing 400014, China; ${ }^{6}$ The Second School of Clinical Medicine, Lanzhou University, Lanzhou 730000, China; ${ }^{7}$ Department of Pediatric, Sichuan Provincial People's Hospital, University of Electronic Science and Technology of China, Chengdu 611731, China; ${ }^{8}$ Chinese Academy of Sciences Sichuan Translational Medicine Research Hospital, Chengdu 610072, China; ${ }^{9}$ School of Public Health, Lanzhou University, Lanzhou 730000, China; ${ }^{10}$ Emergency and Critical Care Center, Department of General Medicine, Department of Research and Medical Education at Kurashiki Central Hospital, Kurashiki, Japan; ${ }^{11}$ Advisory Committee in Cochrane Japan, Tokyo, Japan; ${ }^{12}$ Department of Preventive Medicine, Korea University College of Medicine, Seoul, Korea; ${ }^{13}$ Korea Cochrane Centre, Seoul, Korea; ${ }^{14}$ Korea Institute of Oriental Medicine, Daejeon, Korea; ${ }^{15}$ University of Science and Technology, Daejeon, Korea; ${ }^{16}$ Lanzhou University,An Affiliate of the Cochrane China Network, Lanzhou 730000, China; ${ }^{17}$ Chinese GRADE Center, Lanzhou 730000, China; ${ }^{18}$ Key Laboratory of Evidence Based Medicine and Knowledge Translation of Gansu Province, Lanzhou University, Lanzhou 730000, China; ${ }^{19}$ Department of Oncology Surgery, The First Hospital of Lanzhou University, Lanzhou 730000, China

Contributions: (I) Conception and design: All authors; (II) Administrative support: Y Chen; (III) Provision of study materials or patients: Q Shi, Q Zhou, X Wang; (IV) Collection and assembly of data: Q Shi, Q Zhou, X Wang, J Liao; (V) Data analysis and interpretation: Q Shi, Q Zhou, X Wang, J Liao, Y Yu, Z Wang, S Lu; (VI) Manuscript writing: All authors; (VII) Final approval of manuscript: All authors.

"These authors contributed equally to this work.

Correspondence to: Quanlin Guan. Department of Oncology Surgery, The First Hospital of Lanzhou University, Lanzhou 730000, China. Email: guanquanlin@163.com; Kehu Yang. The First School of Clinical Medicine, Evidence-based Medicine Center, School of Basic Medical Sciences, Lanzhou University, Lanzhou 730000, China. Email: kehuyangebm2006@126.com; Qubei Li. Department of the Respiratory Medicine, Children’s Hospital of Chongqing Medical University, Chongqing 400014, China. Email: liqubei@126.com.
\end{abstract}

Background: The COVID-19 outbreak presents a new, life-threatening disease. Our aim was to assess the potential effectiveness and safety of antiviral agents for COVID-19 in children.

Methods: Electronic databases (MEDLINE, Embase, Web of Science, the Cochrane library, CBM, CNKI, and Wanfang Data) from their inception to March 31, 2020 were searched for randomized controlled trials (RCTs), clinical controlled trials and cohort studies of interventions with antiviral agents for children (less than 18 years of age) with COVID-19.

Results: A total of 23 studies with 6,008 patients were included. There was no direct evidence and all of evidence were indirect. The risks of bias in all studies were moderate to high in general. The effectiveness and safety of antiviral agents for children with COVID-19 is uncertain: For adults with COVID-19, lopinavir/ritonavir had no effect on mortality [risk ratio $(\mathrm{RR})=0.77 ; 95 \%$ confidence interval $(\mathrm{CI}), 0.45$ to 1.30$]$. Arbidol and hydroxychloroquine (HCQ) had no benefit on probability of negative PCR test (RR $=1.27 ; 95 \% \mathrm{CI}, 0.93$ to $1.73 ; \mathrm{RR}=0.93 ; 95 \% \mathrm{CI}, 0.73$ to 1.18 ) respectively. For adults with SARS, interferon was associated with reduced corticosteroid dose [weighted mean difference (WMD) $=-0.14 \mathrm{~g} ; 95 \% \mathrm{CI}$, -0.21 to -0.07 ] but had no effect on mortality ( $\mathrm{RR}=0.72 ; 95 \% \mathrm{CI}, 0.28$ to 1.88 ); ribavirin did not reduce mortality ( $\mathrm{RR}=0.68 ; 95 \% \mathrm{CI}, 0.43$ to 1.06 ) and was associated with high risk of severe adverse reactions; and 
oseltamivir had no effect on mortality ( $R R=0.87 ; 95 \%$ CI, 0.55 to 1.38). Ribavirin combined with interferon was also not effective in adults with MERS and associated with adverse reactions.

Conclusions: There is no evidence showing the effectiveness of antiviral agents for children with COVID-19, and the clinical efficacy of existing antiviral agents is still uncertain. We do not suggest clinical routine use of antivirals for COVID-19 in children, with the exception of clinical trials.

Keywords: Antiviral agents; children; COVID-19; meta-analysis; rapid review

Submitted Apr 13, 2020. Accepted for publication Apr 26, 2020.

doi: 10.21037/atm-20-3301

View this article at: http://dx.doi.org/10.21037/atm-20-3301

\section{Introduction}

A novel coronavirus, later named as severe acute respiratory syndrome coronavirus 2 (SARS-CoV-2), was first detected on December 8, 2019, when several cases of pneumonia of unknown etiology were reported in China (1-3). Due to the rapidly increasing numbers of infections and deaths, World Health Organization (WHO) subsequently declared the outbreak as a Public Health Emergency of International Concern (PHEIC) on January 30, 2019 and officially named the disease as "Corona Virus Disease hyphen one nine" (COVID-19) on February 11, 2020 (4-6). As of April 12, a total of $1,696,588$ confirmed cases had been reported in more than 200 countries, and the number of cases was still rapidly increasing, creating global alarm and concerns about the impact on health care and economy of the affected areas (7). On February 28, WHO increased the level of risk of spread and impact of COVID-19 on the global level to very high and declared COVID-19 as a global pandemic on March 11, 2020 (8). Currently, there is so far no effective treatment or vaccine against the SARS-CoV-2.

At present, guidelines suggest that the use of lopinavir/ ritonavir (LPV/r), interferon (IFN) and chloroquine may help to some extent against COVID-19 in adults (9-11). Seven recently published systematic or rapid reviews suggested that $\mathrm{LPV} / \mathrm{r}$, IFN, chloroquine and hydroxychloroquine (HCQ) can be used as an experimental therapy for COVID-19 in adults as the initial treatment (12-18), but the effectiveness and safety of other antiviral agents [such as ribavirin (RBV), remdesivir and oseltamivir] is uncertain. Although the course of COVID-19 is usually milder in children than adults, children with undeveloped immune system, such as the youngest case confirmed only 30 hours after birth, are at substantial risk of severe infection (19). Antiviral therapy against SARS-CoV-2 in children is therefore urgently needed, but so far the evidence and literature on the topic remain limited $(20,21)$.

The objective of this rapid review is to perform a comprehensive literature search and summarize the current evidence on effectiveness and safety of antiviral agents for children with COVID-19. The findings will provide evidence for the development of guideline and the clinical treatment of children with COVID-19. We presented the following article in accordance with the PRISMA reporting checklist (available at http://dx.doi.org/10.21037/atm-203301) (22).

\section{Methods}

\section{Search strategy}

Two researchers (Q Shi and X Wang) searched the following electronic databases from their inception until March 31, 2020: MEDLINE (via PubMed), Embase, Web of Science, the Cochrane library, China Biology Medicine (CBM), China National Knowledge Infrastructure (CNKI), and Wanfang Data (23). We also searched three clinical trial registry platforms [the WHO Clinical Trials Registry Platform, US National Institutes of Health Trials Register and the International Standard Randomized Controlled Trial Number (ISRCTN) Register], Google Scholar, the official websites of WHO and Centers for Disease Control (CDC), and the preprint platforms BioRxiv, MedRxiv, and SSRN. In addition, we searched the reference lists of the identified systematic reviews for further potential studies. Finally, we contacted experts in the field to identify studies that may have been missed.

The search strategy was also peer reviewed by an external specialist. We systematically searched by combining the MeSH and free words. The keywords and terms in the MEDLINE including "COVID-19", "SARSCoV-2", "Novel coronavirus", "2019-novel coronavirus", 
"2019-nCoV", "antiviral agents", "antiviral"”, "ribavirin", "interferon", "oseltamivir", "remdesivir", "lopinavir", "ritonavir", "LPV/r" and their derivatives. The details of the search strategy can be found in the Supplementary Material I.

\section{Inclusion and exclusion criteria}

We primarily searched for studies on children less than 18 years of age diagnosed with COVID-19. We made no restrictions on gender, race, or geographical location or setting. COVID-19 was defined according to the WHO interim guidance (24). If direct evidence on children was unavailable, we also searched indirect evidence from COVID-19 in adults, or from children or adults infected with severe acute respiratory syndrome coronavirus (SARS$\mathrm{CoV}$ ) and Middle East respiratory syndrome coronavirus (MERS-CoV) which have similar gene sequences with SARS-CoV-2.

We included all randomized controlled trials (RCTs), clinical controlled trials (CCTs) and cohort studies that compared the effectiveness and safety of antiviral agents (including but not limited to IFN, oseltamivir, LPV/r, RBV, HCQ and remdesivir) with placebo, or comparing the combination of antiviral agents and symptomatic treatment with symptomatic treatment alone. Studies comparing different types and different administration mode of antiviral agents were also included. In vitro studies, animal experiments and basic researches were excluded. Duplicates, articles written in languages other than English or Chinese, conference abstracts and studies where full text could not be retrieved or data were missing were also excluded.

The primary outcomes were mortality and the risk of serious adverse effects (defined as hemolytic anemia, bradycardia and other side effects on cardiovascular system and drug-induced liver injury). The secondary outcomes included the probability of negative PCR test (defined as the rate of negative PCR of SARS-CoV-2 after discharge from the hospital or after receiving antiviral agents which differed in studies), mean or reduction in the dose of corticosteroids, remission of the main clinical symptoms, risk of Acute Respiratory Distress Syndrome (ARDS), duration of disease [defined as the duration (in days) of total stay from symptom onset to recovery], probability of admission to intensive care unit (ICU) and other adverse reactions.

\section{Study selection}

After eliminating duplicates, two researchers (Q Shi and X Wang) independently screened first the titles and abstracts, and then the full-texts of potentially relevant articles, using pre-defined criteria. The specific bibliographic software EndNote was used, and discrepancies were discussed, or solved with a third researcher (Q Zhou). The reasons for exclusion of ineligible studies were recorded. The process of study selection was documented using a PRISMA flow diagram (22).

\section{Data extraction}

Four researchers (Q Shi, X Wang, Q Zhou and J Liao) extracted data independently in pairs with a pre-determined form, and disagreements were resolved by consensus. We extracted the following data: (I) basic information; (II) participants: baseline characteristics and inclusion/exclusion criteria; (III) details of the intervention and control strategies; and (IV) outcomes [for dichotomous data, the number of events and total participants in per group; for continuous data, means, standard deviations (SD), and the number of total participants in per group].

\section{Risk of bias assessment}

Two researchers (Y Yu and Z Wang) independently assessed the potential bias in each included study. Discrepancies were resolved by discussion and consensus to a third researcher (S Lu). For RCTs we used the Cochrane Risk-of-Bias (RoB) assessment tool consisting seven domains: random sequence generation, allocation concealment, blinding of participants and personnel, blinding of outcome assessment, incomplete outcome data, selective outcome reporting, and other bias (25). We graded each potential source of bias as "Low", "Unclear" or "High". For included CCTs, we used ROBINS-I tool (26), which consists of seven domains: bias due to confounding, bias in selection of participants, bias in classification of interventions, bias due to departures from intended interventions, bias due to missing data, bias in outcome measurement, and bias in selective reporting. The risk of each type of bias was graded as "Low", "Moderate", "Serious", "Critical", and "No information". For both RoB and ROBINS-I, the overall risk of bias within each study was based on the results of all the individual domains. 
For cohort studies, we used Newcastle-Ottawa Scale (NOS) consisting of three domains (selection of exposure, comparability and assessment of outcome) (27). The maximum score was nine, and scores of seven or more was graded as high quality while less than seven scores as low quality.

\section{Data synthesis}

We performed meta-analyses of outcomes for which the data that were sufficiently compatible. For outcomes with too heterogeneous data, a qualitative synthesis was done. We processed the data according to the Cochrane Handbook by using a random-effects model (28). For dichotomous data, we calculated risk ratios (RR) with $95 \%$ confidence intervals (CI); for continuous data, we calculated weighted mean difference (WMD) with 95\% CI. Two-sided $\mathrm{P}$ values $<0.05$ were considered statistically significant (29). Analyses were performed by Stata 14 software (Stata Corp LLC).

For missing SDs, standard errors (SE) were converted to SDs when SE was presented, and if both were missing, we estimated SDs from P values or $95 \%$ CI. For missing means, we estimated them from interquartile ranges and medians (30). Statistical heterogeneity was assessed using the chi-square and the $I^{2}$ statistic, with $\mathrm{P}<0.10$ was considered to be consistent with statistically significant heterogeneity and $I^{2}$ statistic $>50 \%$ indicating substantial heterogeneity (29). If we detected heterogeneity, we performed subgroup analyses (route, dose, frequency or administration of antivirals) or sensitivity analyses (excluding studies with low-quality or high risk of bias; excluding studies in which mean or SD, or both of them were imputed for missing data) to explore the reasons. Publication bias was assessed by examining the symmetry of the funnel-plot.

\section{Quality of the evidence assessment}

We assessed the quality of evidence using the Grading of Recommendations Assessment, Development and Evaluation (GRADE) approach, and classified the evidence quality as "high", "moderate", "low" and "very low" $(31,32)$. We also produced "Summary of Findings" tables. Direct evidence from RCTs starts at high quality, and evidence from observational studies at low quality. In the next step, the quality can be downgraded for five different reasons (study limitations, consistency of effect, imprecision, indirectness, and publication bias) and upgraded for three reasons (large magnitude of effect, dose-response relation and plausible confounders or biases).

\section{Results}

\section{Study and patient characteristics}

We identified 4,095 references from the databases, and six records from additional searches. A total of 1,216 records were excluded as duplicates, after screening for titles, abstract and full texts, no direct evidence for children with COVID-19 was found. Finally, a total of 23 studies (six RCTs and 17 cohort studies) with 6,008 patients of indirect evidence were included (Figure 1) (33-55). These studies were published between 2003 and 2020 and the sample size ranged from 22 to 1,701, of which, seven studies were on COVID-19, 13 studies on SARS and three studies on MERS. Another study of Cai 2020 was found but in temporary removal condition, therefore it was not included (56).

The risk of bias in the included three RCTs were high, as they did not perform allocation concealment and blindness for patients and clinicians. The other three RCTs had low risk of bias $(n=3)$. More than half of the cohort studies $(n=9)$ had a high risk of bias, the main reasons were being the lack of controlling for important factors that would influence the primary study results, lack of long enough follow-up for outcomes to occur, and inadequate outcome ascertainment. Study characteristics and risk of bias are illustrated in Table 1.

\section{Efficacy and safety of existing antiviral agents}

The results of the meta-analysis for each type of antiviral agent are shown in Table 2. The details of primary data from each retrieved study can be found in http://cdn. amegroups.cn/static/application/c4d76b20b0c53e $8 \mathrm{de} 0 \mathrm{~d} 2$ 9c7063716239/10.21037.atm-20-3301-1.pdf. The details of GRADE for each outcome can be found in http://cdn. amegroups.cn/static/application/44c36ea9fb6d4e0223b539 633be74bdd/10.21037.atm-20-3301-2.pdf. Due to the small number of studies for each outcome, we were unable to evaluate publication bias.

\section{Lopinavir/ritonavir}

Three studies (two RCTs and one cohort study) with a total of 327 patients (33-35) reported the effectiveness and safety of LPV/r in adult patients with COVID-19. There was no statistically significant difference in the mortality 

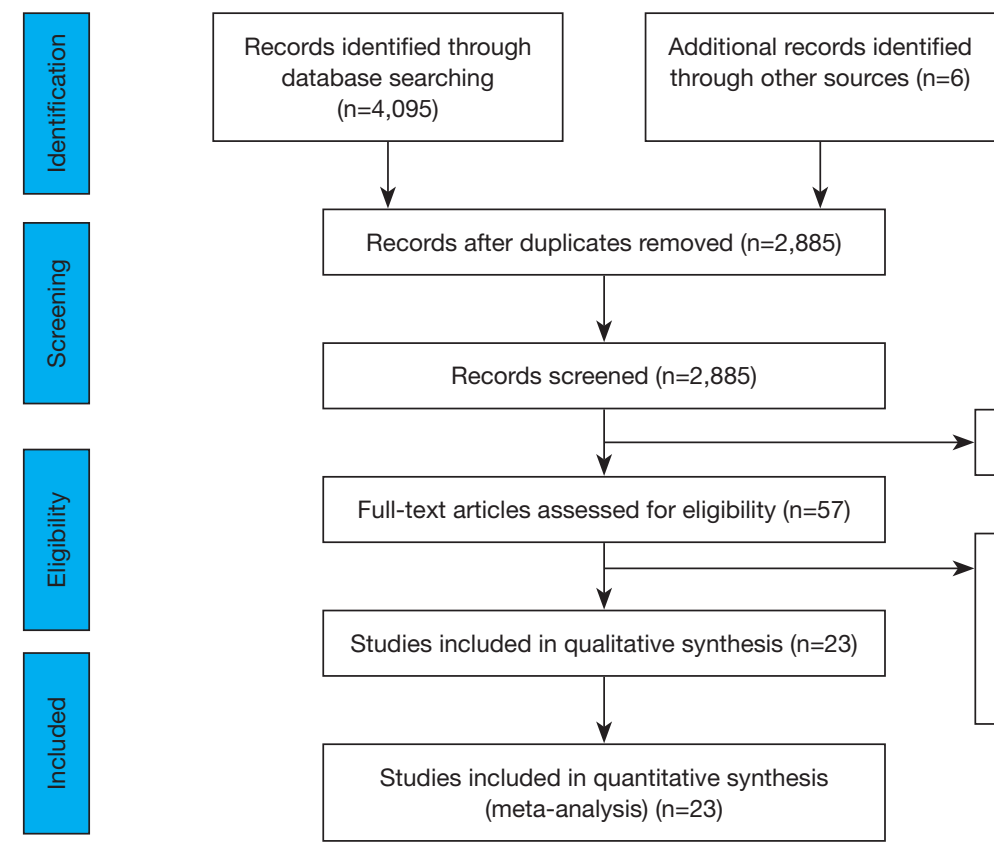

Records excluded $(n=2,828)$

Full-text articles excluded $(n=34)$

- Full-texts cannot be retrieved $(n=2)$

- Case reports/series $(n=5)$

- Reviews and protocols $(n=24)$

- Conference article $(n=3)$

Figure 1 Flow diagram of the literature search.

( $\mathrm{RR}=0.77 ; 95 \% \mathrm{CI}, 0.45$ to 1.30 , low-quality evidence, Figure 2) and probability of negative PCR test ( $\mathrm{RR}=0.98$; $95 \% \mathrm{CI}, 0.82$ to 1.18 , very low-quality evidence) between the intervention and control groups. There was also no statistically significant difference in the incidence of adverse reactions ( $\mathrm{RR}=1.24 ; 95 \% \mathrm{CI}, 0.67$ to 2.28 , very low-quality evidence) and serious adverse reactions ( $R R=0.62 ; 95 \%$ CI, 0.38 to 1.01 , moderate-quality evidence) between the two groups, of which, the most common side effects were gastrointestinal reaction (including nausea and vomiting, diarrhea and abnormal liver function).

Two cohort studies with a total of 830 patients $(36,37)$ reported the effectiveness and safety of $\mathrm{LPV} / \mathrm{r}$ in adult patients of SARS. The results showed that LPV/r therapy decreased the risk of death ( $\mathrm{RR}=0.16 ; 95 \% \mathrm{CI}, 0.03$ to 0.77 , low-quality evidence, Figure 2$)$ and ARDS (RR $=0.11$; $95 \% \mathrm{CI}, 0.02$ to 0.77 , very low-quality evidence) compared with the control group. However, no statistically significant difference was found in the dose of corticosteroids (WMD $=-0.82 \mathrm{~g} ; 95 \% \mathrm{CI},-2.03$ to 0.40 ) with considerable heterogeneity of the I-squared was $86.4 \%$, because both means and SDs of the two studies were imputed from missing data. In addition, patients in the LPV/r group were more often nosocomially infected $(\mathrm{RR}=0.05 ; 95 \% \mathrm{CI}, 0.00$ to 0.75 ), and had a higher risk of adverse reactions such as diarrhea ( $\mathrm{RR}=0.39 ; 95 \% \mathrm{CI}, 0.23$ to 0.69 ) or recurrent fever ( $R R=0.65 ; 95 \% \mathrm{CI}, 0.43$ to 0.98$)$. The overall quality of evidence was very low.

\section{Arbidol}

Three studies (one RCT and two cohort studies) with a total of 138 patients $(34,35,38)$ reported the effectiveness and safety of arbidol in adult patients of COVID-19. There was no statistically significant difference in the probability of having a negative PCR result ( $\mathrm{RR}=1.27$; 95\% CI, 0.93 to 1.73 ), probability of radiographic abnormalities remission $(\mathrm{RR}=1.23 ; 95 \% \mathrm{CI}, 0.63$ to 2.40$)$ and duration of disease (WMD $=-1.70$ days; $95 \% \mathrm{CI},-3.28$ to -0.12 ) between patients with arbidol therapy and control group. Because of the large heterogeneity in the radiographic abnormalities remission, we performed a subgroup analysis of study design, and we still found no significant association in neither cohort studies ( $\mathrm{RR}=1.58 ; 95 \% \mathrm{CI}, 0.97$ to 2.59 ) nor RCTs (RR $=0.71 ; 95 \%$ CI, 0.47 to 1.06 ). There was also no statistically significant difference in the incidence of adverse reactions ( $\mathrm{RR}=1.06$; $95 \% \mathrm{CI}, 0.25$ to 4.43 ) between the two groups. The overall quality of evidence was very low. 


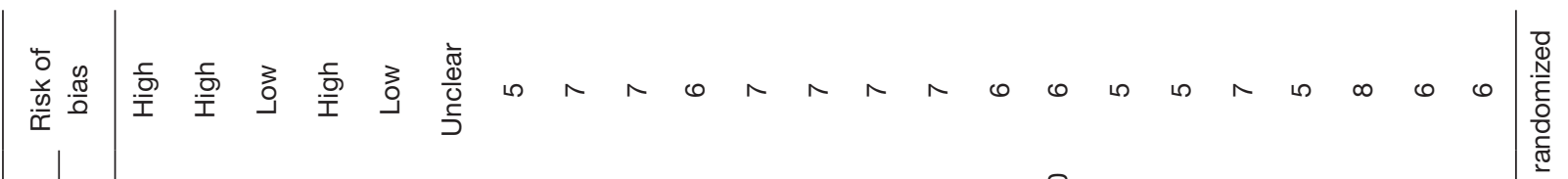

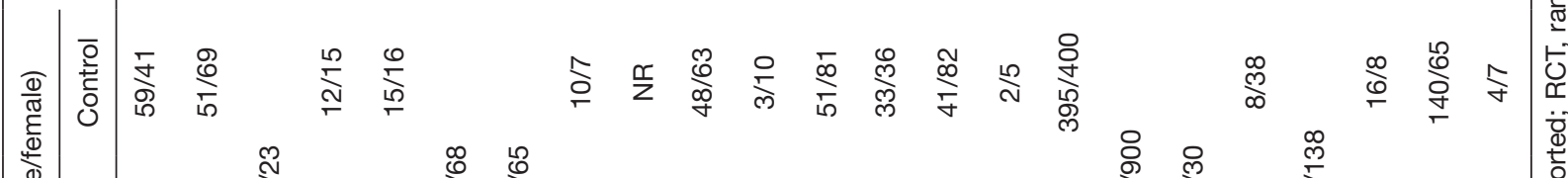

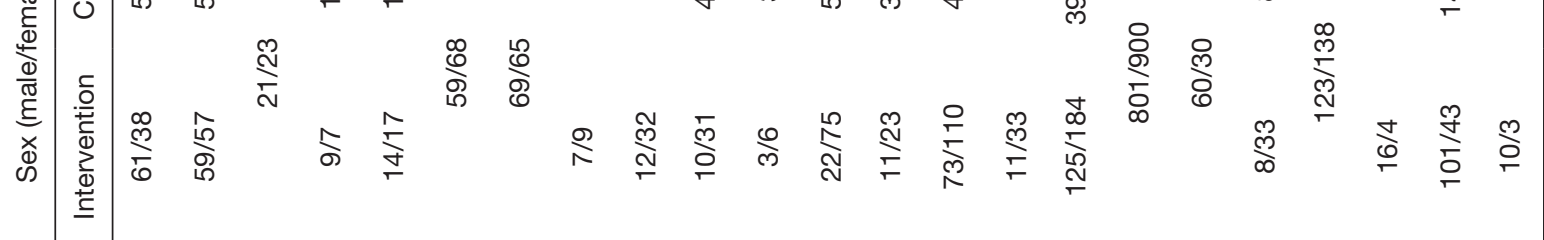

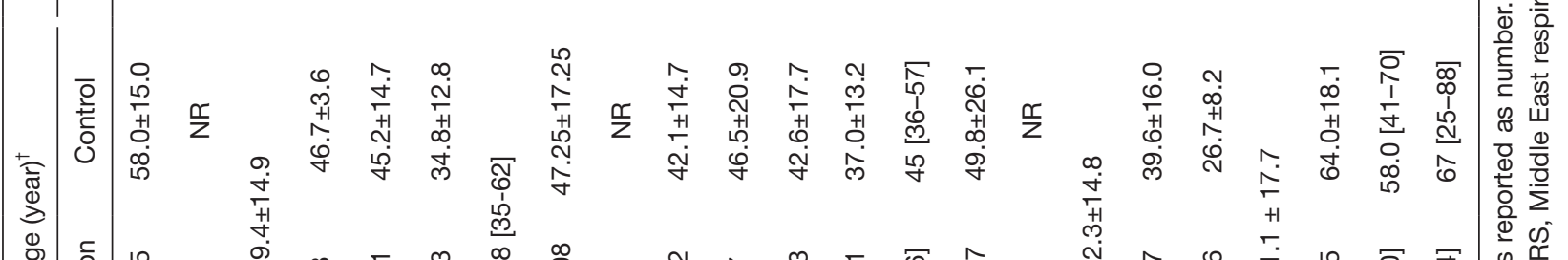

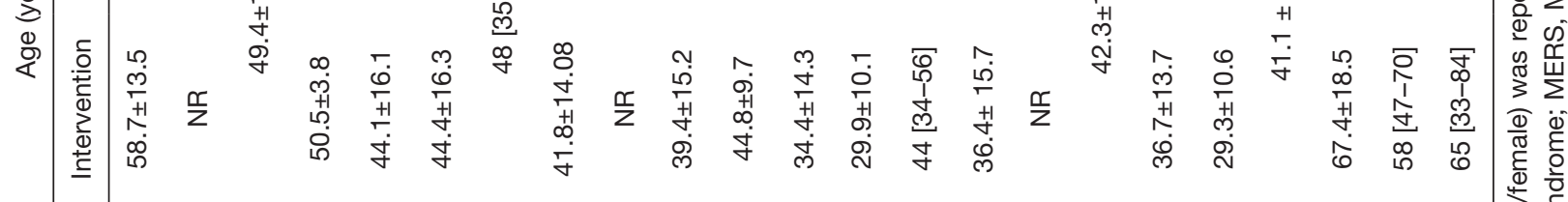

$$
\begin{aligned}
& \text { 旁 웅 운 } \\
& \text { }
\end{aligned}
$$

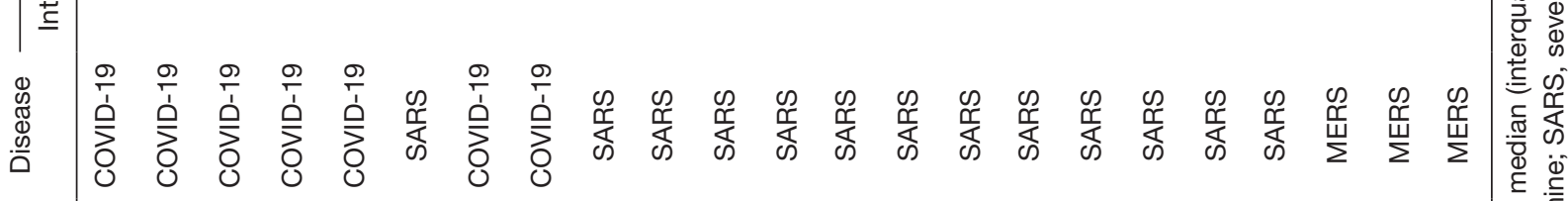

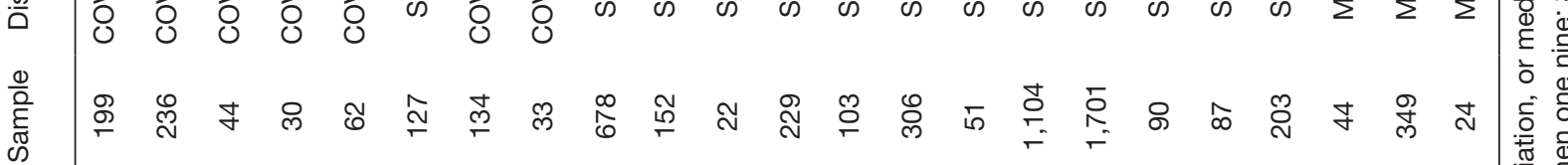

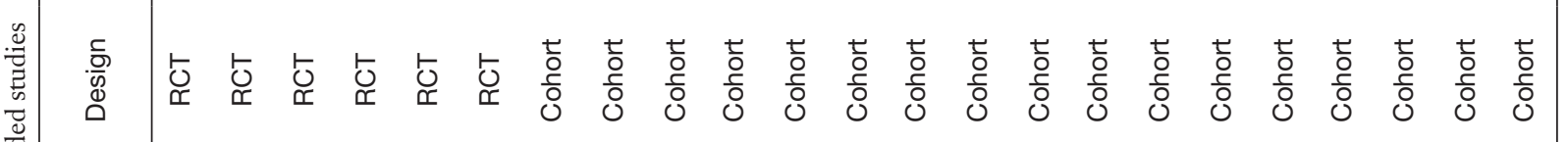

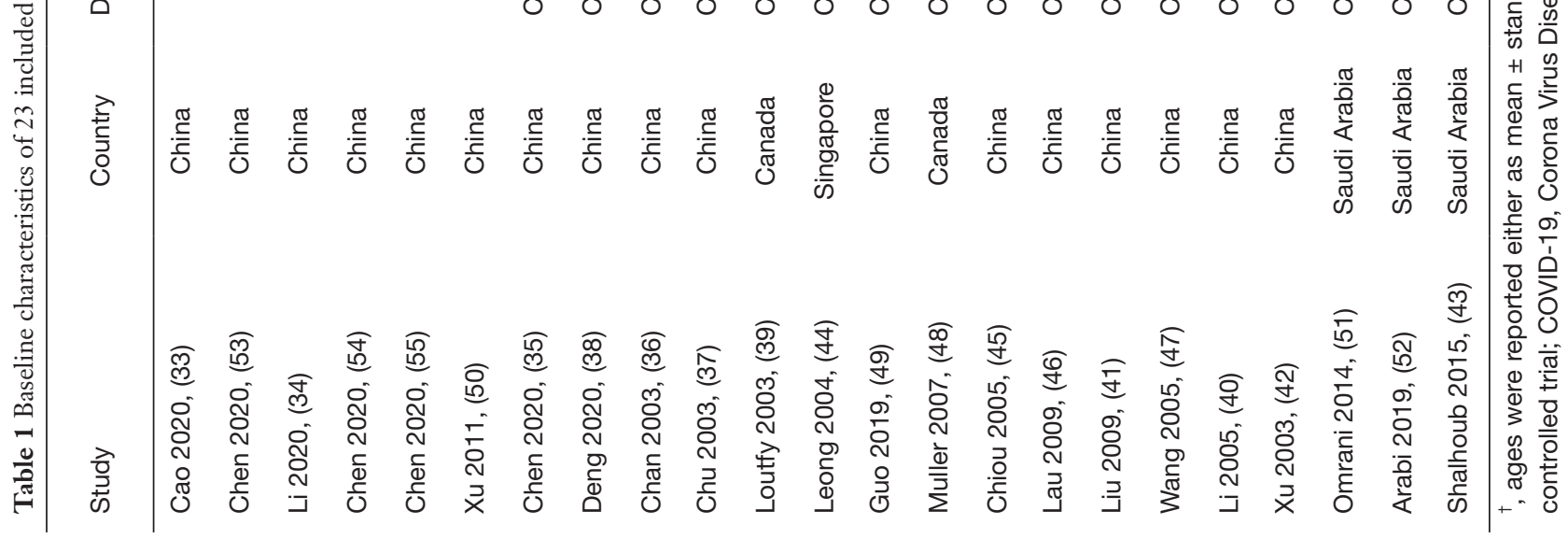


Table 2 Summary of evidence for the effectiveness and safety of antiviral agents

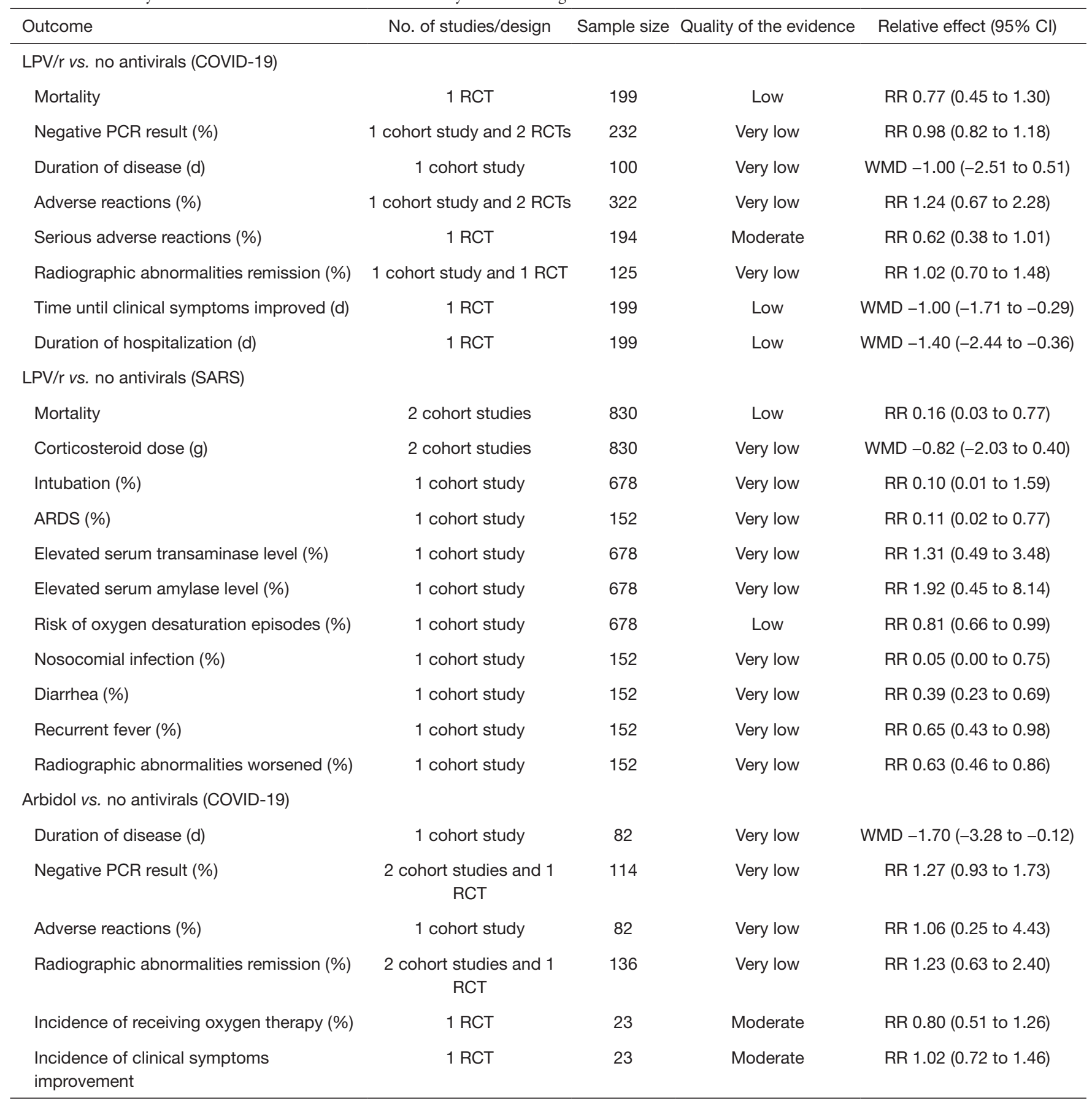

Table 2 (continued) 
Table 2 (continued)

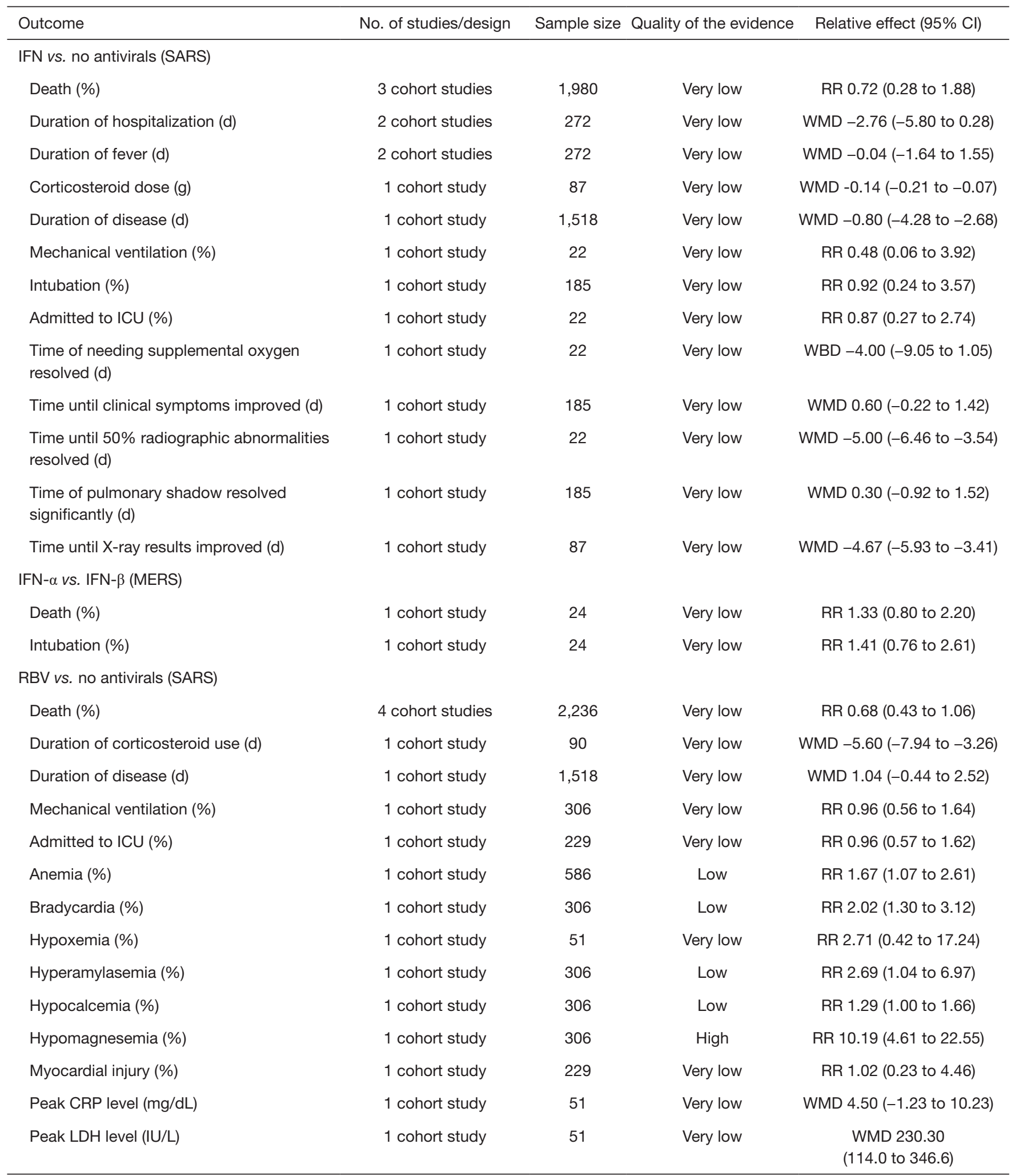

Table 2 (continued) 
Table 2 (continued)

\begin{tabular}{|c|c|c|c|c|}
\hline Outcome & No. of studies/design & Sample size & Quality of the evidence & Relative effect $(95 \% \mathrm{Cl})$ \\
\hline \multicolumn{5}{|l|}{ Oseltamivir vs. no antivirals (SARS) } \\
\hline Death (\%) & 3 cohort studies & 1,887 & Very low & RR 0.87 (0.55 to 1.38$)$ \\
\hline Mechanical ventilation (\%) & 1 cohort study & 103 & Low & RR 2.67 (1.73 to 4.12 ) \\
\hline Intubation (\%) & 1 cohort study & 83 & Very low & RR 0.50 (0.03 to 9.19) \\
\hline ARDS (\%) & 1 cohort study & 103 & Very low & RR 1.29 (0.55 to 3.03$)$ \\
\hline Duration of hospitalization (d) & 1 cohort study & 83 & Very low & $\begin{array}{c}\text { WMD }-7.60 \\
(-10.49 \text { to }-4.71)\end{array}$ \\
\hline Time until clinical symptoms improved (d) & 1 cohort study & 83 & Very low & WMD 2.60 (-0.25 to 5.45$)$ \\
\hline Duration of fever (d) & 1 cohort study & 83 & Very low & WMD 2.60 (0.50 to 4.70$)$ \\
\hline \multicolumn{5}{|c|}{ Oseltamivir (early use alone) vs. oseltamivir (use alone) (SARS) } \\
\hline Death (\%) & 1 cohort study & 127 & Low & RR 1.62 (0.33 to 8.05$)$ \\
\hline ARDS (\%) & 1 cohort study & 127 & Low & RR 2.60 (0.30 to 22.57 ) \\
\hline Duration of disease $(d)$ & 1 cohort study & 127 & Low & WMD $-2.50(-7.45$ to 2,45$)$ \\
\hline Duration of fever (d) & 1 cohort study & 127 & Low & WMD $-0.90(-1.91$ to 0.11$)$ \\
\hline \multicolumn{5}{|l|}{ RBV plus IFN vs. no antivirals (MERS) } \\
\hline Death (\%) & 2 cohort studies & 393 & Very low & RR 1.04 (0.74 to 1.46$)$ \\
\hline Invasive ventilation (\%) & 2 cohort studies & 393 & Very low & RR 1.05 (0.97 to 1.13$)$ \\
\hline \multicolumn{5}{|l|}{ Favipiravir vs. Arbidol (COVID-19) } \\
\hline Rate of clinical recovery of day $7(\%)$ & $1 \mathrm{RCT}$ & 236 & Low & RR 1.18 (0.95 to 1.48$)$ \\
\hline Adverse reactions (\%) & $1 \mathrm{RCT}$ & 236 & Low & RR 1.37 (0.90 to 2.08) \\
\hline Dyspnea after taking medicine (\%) & $1 \mathrm{RCT}$ & 236 & Low & RR 0.30 (0.10 to 0.87$)$ \\
\hline Respiratory failure (\%) & $1 \mathrm{RCT}$ & 236 & Low & RR 1.03 (0.26 to 4.04$)$ \\
\hline \multicolumn{5}{|l|}{ HCQ vs. none (COVID-19) } \\
\hline Negative PCR result (\%) & $1 \mathrm{RCT}$ & 30 & Low & RR 0.93 (0.73 to 1.18) \\
\hline Radiographic abnormalities remission (\%) & $1 \mathrm{RCT}$ & 62 & Low & RR 1.47 (1.02 to 2.11$)$ \\
\hline Duration of fever (d) & 2 RCTs & 69 & Low & WMD $-0.90(-1.48$ to -0.31$)$ \\
\hline Time until negative PCR result (d) & $1 \mathrm{RCT}$ & 30 & Low & WMD 2.34 (-1.19 to 5.87$)$ \\
\hline Adverse reactions (\%) & $2 \mathrm{RCTs}$ & 92 & Low & RR 1.65 (0.50 to 5.50$)$ \\
\hline
\end{tabular}

LPV/r, lopinavir/ritonavir; IFN, interferon; RBV, ribavirin; HCQ, hydroxychloroquine; Cl, confidence interval; RR, risk ratio; WMD, weighted mean difference; RCT: randomized controlled trial; COVID-19, Corona Virus Disease hyphen one nine; SARS, severe acute respiratory syndrome; MERS, Middle East respiratory syndrome ARDS, Acute respiratory distress syndrome; PCR, polymerase chain reaction; ICU, intensive care unit; CRP, C-reactive protein; LDH, lactate dehydrogenase. 


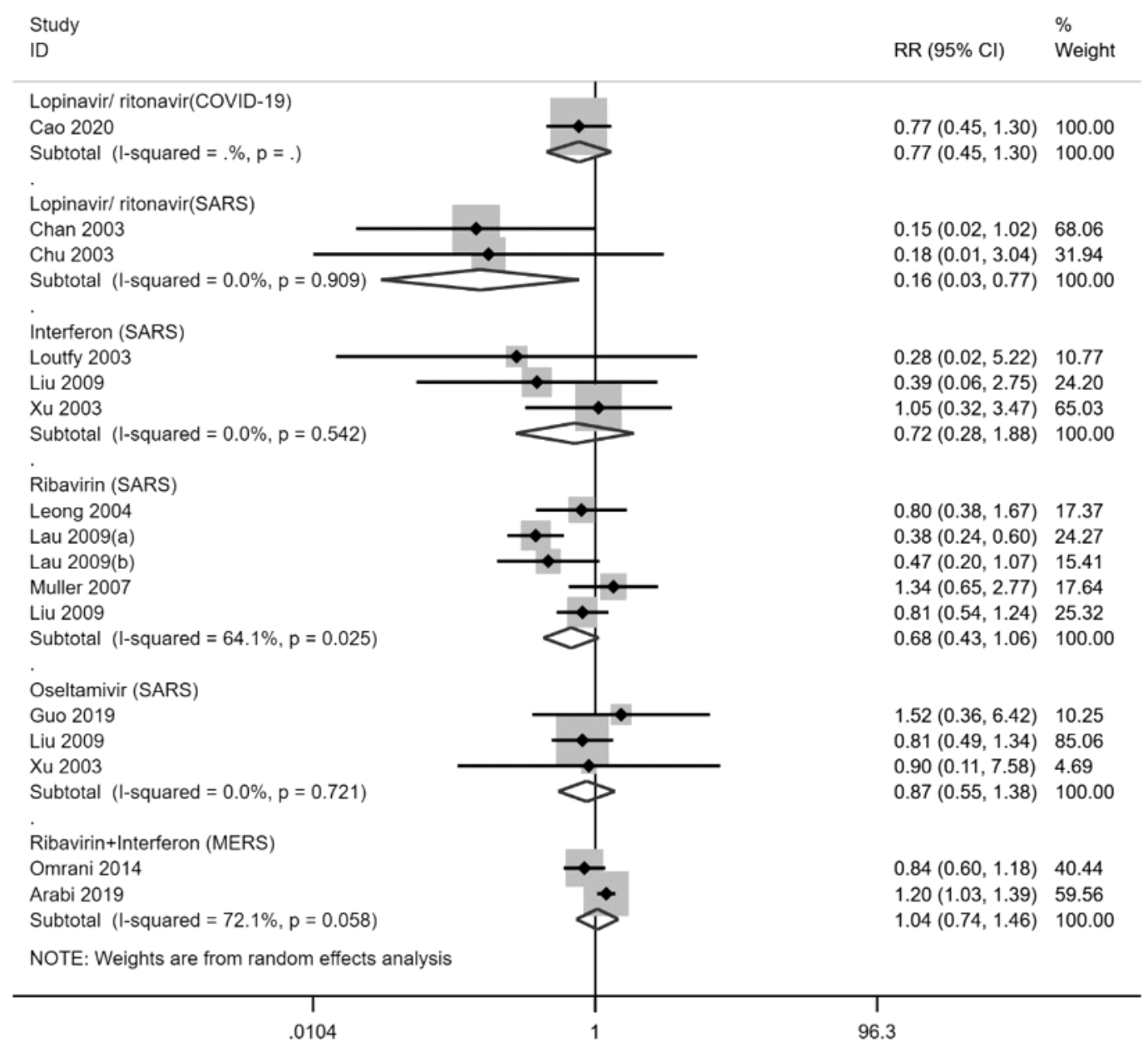

Figure 2 Forest plot of mortality for included studies comparing antivirals with no antivirals.

\section{Interferon}

Four cohort studies with a total of 2,013 patients (39-42) reported the effectiveness and safety of intramuscular or subcutaneous injection of IFN in adult patients with SARS. The results showed that IFN therapy decreased the dose of corticosteroids dose (WMD $=-0.14 \mathrm{~g} ; 95 \% \mathrm{CI},-0.21$ to -0.07$)$ and promoted the remission of radiographic abnormalities. No statistically significant difference was found in mortality ( $\mathrm{RR}=0.72 ; 95 \% \mathrm{CI}, 0.28$ to 1.88 , Figure 2). No obvious adverse reactions were reported in any of the above four studies. The quality of evidence was very low.

One cohort study with a total of 24 patients (43) compared the effectiveness of different types of IFN in adult patients with SARS. The results showed that there was no statistically significant difference in the risk of death $(\mathrm{RR}=1.33$; $95 \%$
CI, 0.80 to 2.20 ) between patients treated with IFN- $\alpha$ and IFN- $\beta$. The quality of evidence was very low.

\section{Ribavirin}

Six cohort studies with a total of 3,481 patients $(41,44-48)$ reported the effectiveness and safety of RBV in adult patients with SARS. The results showed that RBV therapy significantly decreased the duration of corticosteroid use $(\mathrm{WMD}=-5.60 \mathrm{~g} ; 95 \% \mathrm{CI},-7.94$ to -3.26 , very low-quality evidence), and increased the duration of disease (WMD $=1.04 \mathrm{~d}$; $95 \% \mathrm{CI},-0.44$ to 2.52 , very low-quality evidence) compared with the control group. There was no statistically difference in the risk of death ( $\mathrm{RR}=0.68 ; 95 \% \mathrm{CI}, 0.43$ to 1.06 , Figure 2). In addition, the use of RBV was associated with an increased risk of adverse reactions, including anemia 
( $\mathrm{RR}=1.67 ; 95 \% \mathrm{CI}, 1.07$ to 2.61 , low-quality evidence), bradycardia ( $\mathrm{RR}=2.02 ; 95 \% \mathrm{CI}, 1.30$ to 3.12 , low-quality evidence), and hypomagnesemia ( $\mathrm{RR}=10.19 ; 95 \% \mathrm{CI}, 4.61$ to 22.55 , high-quality evidence).

\section{Oseltamivir}

Three cohort studies with a total of 2,007 patients $(41,42,49)$ reported the effectiveness and safety of oseltamivir in adult patients with SARS. The results showed that there was no statistically significant difference in the risk of death (RR $=0.87 ; 95 \%$ CI, 0.55 to 1.38 , Figure 2) between oseltamivir therapy and the control group. The use of oseltamivir prolonged the duration of disease (WMD $=3.91$ days; $95 \%$ CI, 2.28 to 5.54, very low-quality evidence) and duration of fever (WMD $=2.60$ days, $95 \% \mathrm{CI}, 0.50$ to 4.70 , very lowquality evidence).

One RCT with a total of 127 patients (50) compared the effectiveness of oseltamivir between early use alone and use alone in adult patients with SARS. The results showed that early use alone was not associated with the risk of death (RR $=1.62 ; 95 \% \mathrm{CI}, 0.33$ to 8.05 ), $\mathrm{ARDS}$ ( $\mathrm{RR}=2.60 ; 95 \% \mathrm{CI}$, 0.30 to 22.57 ) or the duration of disease (WMD $=-2.50$ days, $95 \%$ CI, -7.45 to 2.45 ). The quality of evidence was low.

\section{Combination of ribavirin and interferon}

Two cohort studies with a total of 393 patients $(51,52)$ reported the effectiveness and safety of a combination of RBV and IFN for adult patients with MERS. The results showed that combination therapy of RBV and IFN could increase the mean reduction in hemoglobin (WMD = $2.18 \mathrm{~g} / \mathrm{L} ; 95 \% \mathrm{CI}, 0.86$ to 3.50 , very low-quality evidence) and the need of blood transfusion ( $\mathrm{RR}=1.42 ; 95 \% \mathrm{CI}, 1.06$ to 1.91 , low-quality evidence). But there was no statistically significant difference in the risk of death ( $R R=1.04 ; 95 \%$ CI, 0.74 to 1.46 , Figure 2) between the two groups.

\section{Favipiravir}

One study of RCT with a total of 236 patients (53) reported the effectiveness and safety of favipiravir for adult patients with COVID-19. The results showed that when comparing to arbidol, favipiravir had lower incidence of dyspnea after taking medicine ( $\mathrm{RR}=0.30 ; 95 \% \mathrm{CI}, 0.10$ to 0.87$)$, but there was no difference in clinical recovery $(\mathrm{RR}=1.18$;
$95 \%$ CI, 0.95 to 1.48 ) or the incidence of adverse reactions ( $\mathrm{RR}=1.37 ; 95 \% \mathrm{CI}, 0.90$ to 2.08 ). The overall quality of evidence was low.

\section{HCQ}

Two studies of RCTs with a total of 92 patients $(54,55)$ reported the effectiveness and safety of HCQ for adult patients with COVID-19. The results showed that HCQ had no benefit on the negative PCR result $(\mathrm{RR}=0.93$; $95 \%$ CI, 0.73 to 1.18 ), but was effective for shortening the duration of fever (WMD $=-0.90$ days, 95\% CI, -1.48 to -0.31 ). In addition, there was no statistically significant difference in the incidence of adverse reactions $(R R=1.65$; 95\% CI, 0.50 to 5.50 ) between HCQ therapy and the control group. The overall quality of evidence was low.

\section{Discussion}

Our rapid review identified a total of 23 studies. No direct evidence for the effectiveness and safety of antiviral agents for children with COVID-19 was available. Based on the analysis of indirect evidence from adult patients with COVID-19, very low to low-quality evidence indicated that LPV/r, arbidol and HCQ were not effective. For adult patients with SARS or MERS, very low to low-quality evidence indicated that LPV/r, IFN, RBV and oseltamivir had no clinical effectiveness on mortality, corticosteroids dose, or other main outcomes. Certain medications, such as LPV/r and RBV, were likely to lead to adverse reactions (such as gastrointestinal reaction, abnormal liver function, anemia, bradycardia, or hypoxemia).

Most viral diseases are self-limiting illnesses that do not require specific antiviral therapy. At present, no antiviral agent has been confirmed to be effective against COVID-19, and vaccination are currently under development, so symptomatic and supportive treatments are crucial. However, children are less likely than adults to have complications or develop into critical conditions, and their clinical manifestations are less atypical, complicating the diagnosis (57-59). Guidelines recommend antiviral agents such as LPV/r, IFN, arbidol and chloroquine to treat COVID-19 in adults, while children (especially critically illness) can be treated reference to the regimen of adults (9-11). Up to now, almost all COVID-19 patients (adults and children) have received antiviral therapy (60). Several 
case reports or series $(61,62)$ have also highlighted the potential efficacy of antivirals in children with SARS-CoV-2 infection and found no obvious adverse reactions, but the number was too small to draw any conclusions. More studies are needed to further evaluate the risks and benefits that antiviral agents may bring.

$\mathrm{LPV} / \mathrm{r}$ is one of the first medications that were taken into clinical practice after the beginning of the COVID-19 outbreak, and it is recommended for treatment of COVID-19 patients in the latest version of China national practice guideline (released on March 4, 2020) without any reference (9). Our rapid review however demonstrates that LPV/r is unlikely to be effective for COVID-19 in adults with numerous obvious adverse reactions, which was the same as recent studies $(63,64)$. Two rapid reviews conducted in $2020(12,13)$ examined that early use of LPV/r can reduce the mortality and steroid dosing in patients with SARS and MERS, and suggested that it could be used as a component for an experimental regimen to treat COVID-19. But no quantitative analysis or evidence grading was performed, and therefore the reliability of the conclusions is questionable. Although LPV/r could reduce the mortality of adult SARS patients, the quality of evidence was low. Therefore, LPV/r should not be recommended in clinical practice guidelines.

The results on other antivirals were similar to those identified in other systematic reviews. Among patients with COVID-19, the use of HCQ was effective for clinical recovery which is the same as published reviews $(17,18)$, but we found HCQ had no benefit on probability of viral load disappearance, this is not the same as previous studies due to the retraction of Philippe 2020 (65). All trials for HCQ included in this study had small sample size to draw robust conclusions. IFN had no benefit on mortality and the effect did not differ between IFN- $\alpha$ and IFN- $\beta$ : the results are in line with another recent rapid review (14). RBV and oseltamivir were not shown effective for treating adults with SARS, and the use of RBV was even related to a high risk of serious side effects, and oseltamivir prolonged the duration of disease. These results were also observed by recent and previous systematic reviews $(15,66,67)$. One study demonstrated that the concentration of RBV required to effectively inhibit the activity of SARS-CoV or MERS$\mathrm{CoV}$ was beyond the clinically acceptable range, so routine use of the drug would have no effect (68). One recent case of COVID-19 in the United States suggested a promising clinical response to remdesivir (69), and study by Wang et al. revealed that remdesivir was highly effective in the control of SARS-CoV-2 in vitro (70), but the evidence quality was low and the newest results of clinical research suggested no significant effect for patients hospitalized of severe COVID-19 (71), and the clinical trials of remdesivir therapy are still ongoing. The outbreak of COVID-19 has imposed a great socioeconomic, public health, and clinical burden on the affected countries and regions, especially for the low-and middle-income countries. Therefore, priority should be given for the research and implementation of agents with promising outcomes.

\section{Strengthens and limitations}

This study is to our knowledge the first systematic and comprehensive rapid review for the effectiveness and safety of antiviral agents in children with COVID-19. It can therefore be considered the best evidence at the moment for the management of COVID-19 in children, and help to respond to the current public health emergency. Our study was also performed and reported in accordance with Cochrane Handbook and PRISMA checklist, and included meta-analyses and grading of evidence to draw quantitative conclusions with scientific and rigorous methods. However, our study had also some limitations: first, this rapid review was unable to identify direct evidence for antiviral use in children with COVID-19 and only summarized the indirect evidence, mainly from adult patients with COVID-19, SARS or MERS. The reported treatment effects should be interpreted with caution due to the lack of high-quality RCTs and direct evidence. Second, due to the heterogeneity of the reviewed studies in terms of the wide range of treatment dosages, frequencies and routes of administration, we were unable to perform a quantitative analysis from these aspects for each antiviral. This is a major obstacle to a clear interpretation of the results of this review. Third, because of the specificity and urgency of PHEIC, our study protocol was not registered on the Prospective Register of Systematic Reviews Platform.

\section{Further suggestions}

We suggest for the further actions on the basis of our study. First, high-quality clinical research should be carried out in a timely and effective manner, following the randomization, control and bind principles of evidencebased medicine, trying to adopt objective and representative outcomes for evaluation, so that unbiased research results can be ensured. Second, health workers need high-quality, 
unbiased and evidence-based recommendations to guide clinical practice. Health workers should accumulate clinical experience and be encouraged to interpret the evidence with professionalism by cooperating with researchers, avoid conflicts of interest, and thus reduce the possibly harmful impact on children with COVID-19. Third, health policy decisions should be made based on the best available evidence and make full use of the limited resources to make decisions that are valid, rational and based on up-to-date scientific knowledge.

\section{Conclusions}

In conclusion, there is no direct evidence for antiviral agents in children with COVID-19 so far. Very low to lowquality indirect evidence indicated that antiviral agents were not effective for reducing mortality, and the effectiveness and safety of antivirals for children with COVID-19 are uncertain. Therefore, we cannot suggest routine use of these agents for the treatment of COVID-19 in children, with the exception of clinical trials after thorough ethical assessment.

\section{Acknowledgments}

We thank Janne Estill, Institute of Global Health of University of Geneva for providing guidance and comments for our review. We thank all the authors for their wonderful collaboration.

Funding: This work was supported by grants from National Clinical Research Center for Child Health and Disorders (Children's Hospital of Chongqing Medical University, Chongqing, China) (grant number NCRCCHD-2020EP-01) to EL; Special Fund for Key Research and Development Projects in Gansu Province in 2020, to YC; The fourth batch of "Special Project of Science and Technology for Emergency Response to COVID-19" of Chongqing Science and Technology Bureau, to EL; Special funding for prevention and control of emergency of COVID-19 from Key Laboratory of Evidence Based Medicine and Knowledge Translation of Gansu Province (grant number No. GSEBMKT-2020YJ01), to YC; The Fundamental Research Funds for the Central Universities (lzujbky-2020-sp14), to YC.

\section{Footnote}

Reporting Checklist: The authors have completed the
PRISMA reporting checklist. Available at http://dx.doi. org/10.21037/atm-20-3301

Conflicts of Interest: All authors have completed the ICMJE uniform disclosure form (available at http://dx.doi. org/10.21037/atm-20-3301). MSL serves as the unpaid editorial board member of Annals of Translational Medicine from Nov 2019 to Oct 2021. The other authors have no conflicts of interest to declare.

Ethical Statement: The authors are accountable for all aspects of the work in ensuring that questions related to the accuracy or integrity of any part of the work are appropriately investigated and resolved.

Open Access Statement: This is an Open Access article distributed in accordance with the Creative Commons Attribution-NonCommercial-NoDerivs 4.0 International License (CC BY-NC-ND 4.0), which permits the noncommercial replication and distribution of the article with the strict proviso that no changes or edits are made and the original work is properly cited (including links to both the formal publication through the relevant DOI and the license). See: https://creativecommons.org/licenses/by-nc-nd/4.0/.

\section{References}

1. Zhu N, Zhang D, Wang W, et al. A novel coronavirus from patients with pneumonia in China, 2019. N Engl J Med 2020;382:727-33.

2. Paules CI, Marston HD, Fauci AS. Coronavirus Infections-More Than Just the Common Cold. JAMA 2020;323:707-8.

3. Huang C, Wang Y, Li X, et al. Clinical features of patients infected with 2019 novel coronavirus in Wuhan, China. Lancet 2020;395:497-506.

4. WHO Director-General's remarks at the media briefing on 2019-nCoV on 11 February 2020. 2020 [cited 2020 Apr 2]. Available online: https://www.who.int/dg/speeches/ detail/who-director-general-s-remarks-at-the-mediabriefing-on-2019-ncov-on-11-february-2020

5. Munster VJ, Koopmans M, van Doremalen N, et al. A novel coronavirus emerging in China-key questions for impact assessment. N Engl J Med 2020;382:692-4.

6. Phelan AL, Katz R, Gostin LO. The Novel Coronavirus Originating in Wuhan, China: Challenges for Global Health Governance. JAMA 2020;323:709-10.

7. World Health Organization (WHO).Coronavirus 
disease 2019 (COVID-19) Situation Report-83. Geneva: WHO; 2020.[cited 2020 Apr 13]. Available online: https://www.who.int/docs/default-source/coronaviruse/ situation-reports/20200412-sitrep-83-covid-19. pdf? sfvrsn=697ce98d_4

8. WHO Director-General's opening remarks at the media briefing on COVID-19 - 28 February 2020 [cited 2020

Apr 2]. Available online: https://www.who.int/dg/speeches/ detail/who-director-general-s-opening-remarks-at-themedia-briefing-on-covid-19---28-february-2020

9. National Health Commission and National Administrative Office of Chinese Tradition Medicine. National Recommendations for Diagnosis and Treatment of pneumonia caused by 2019-nCoV (the 7th edition). [cited 2020 Apr 2]. Available online: http://www.nhc.gov. cn/yzygj/s7653p/202003/46c9294a7dfe4cef80dc7f5912 eb1989.shtml

10. Jin YH, Cai L, Cheng Z, et al. A rapid advice guideline for the diagnosis and treatment of 2019 novel coronavirus (2019-nCoV) infected pneumonia (standard version). Mil Med Res 2020;7:4.

11. The Society of Pediatrics, Chinese Medical Association. Recommendations for Diagnosis and Prevention of 2019 novel Coronavirus Infection in Children (Trial version 1). Chin J Pediatr 2020;58:169-74.

12. Jiang H, Deng H, Wang Y, et al. The possibility of using Lopinave/Litonawe (LPV/r) as treatment for novel coronavirus 2019-nCov pneumonia: a quick systematic review based on earlier coronavirus clinical studies. Chin J Emerg Med 2020;29:182-6.

13. Yao TT, Qian J, Zhu W, et al. A Systematic Review of Lopinavir Therapy for SARS Coronavirus and MERS Coronavirus-A Possible Reference for Coronavirus Disease-19 Treatment Option. J Med Virol 2020. [Epub ahead of print].

14. Chen M, Tong R, Bian Y, et al. Evidence-based Rapid Review on Possibility of Treatment of 2019-nCoV with Subcutaneous $\alpha$-interferon. Her Med 2020.

15. Luo T, Na Y, Tan L, et al. The Possibility of Ribavirin as in the Treatment of the Coronavirus Disease 2019: A Systematic Review. China Pharmaceuticals 2020;29(5).

16. Hong D, Wang R, Ye Z, et al. The effect of interferon combined with ribavirin on the risk of death in patients with Severe Coronavirus Infection: A Systematic Review based on previous MERS-CoV studies. Chin J Hosp Pharm 2020. [Epub ahead of print].

17. Krishan MK, Aanandita K. Role of Chloroquine and Hydroxychloroquine in the Treatment of COVID-19
Infection- A Systematic Literature Review. 2020; MedRxiv 2020. [cited 2020 Apr 2]. Available online: https://www. medrxiv.org/content/10.1101/2020.03.24.20042366v1

18. Cortegiani A, Ingoglia G, Ippolito M, et al. A systematic review on the efficacy and safety of chloroquine for the treatment of COVID-19. J Crit Care 2020. [Epub ahead of print].

19. Zeng LK, Tao X, Yuan W, et al. First case of neonate infected with novel coronavirus pneumonia in China. Zhonghua Er Ke Za Zhi 2020;58:E009.

20. Coronavirus latest: children are as susceptible as adults, study suggests. Nature 2020; [cited 2020 Apr 2]. Available online: https://www.nature.com/articles/d41586-02000154-w

21. Wei M, Yuan J, Liu Y, et al. Novel Coronavirus Infection in Hospitalized Infants Under 1 Year of Age in China. JAMA 2020. [Epub ahead of print].

22. Moher D, Liberati A, Tetzlaff J, et al. PRISMA Group. Preferred Reporting Items for Systematic Reviews and Meta-analyses: the PRISMA statement. BMJ 2009;339:b2535.

23. Li, L, Tian J, Tian H, et al. Network meta-analyses could be improved by searching more sources and by involving a librarian. J Clin Epidemiol 2014;67:1001-7.

24. World Health Organization. Clinical management of severe acute respiratory infection when Novel coronavirus $(\mathrm{nCoV})$ infection is suspected: Interim Guidance. Jan 28, 2020. [cited 2020 Apr 2]. Available online: https:// www.who.int/internal-publications-detail/clinicalmanagementof-severe-acute-respiratory-infection-whennovel-coronavirus-(ncov)-infection-is-suspected

25. Higgins JP, Altman DG, Gotzsche PC, et al. The Cochrane Collaboration's tool for assessing risk of bias in randomised trials. BMJ 2011;343:d5928.

26. Sterne JAC, Hernán MA, Reeves BC, et al. ROBINS-I: a tool for assessing risk of bias in non-randomised studies of interventions. BMJ 2016;355:i4919.

27. Stang A. Critical evaluation of the Newcastle-Ottawa scale for the assessment of the quality of nonrandomized studies in meta analyses. Eur J Epidemiol 2010;25:603-5.

28. DerSimonian R, Kacker R. Random-effects model for meta-analysis of clinical trials: an update. Contemp Clin Trials 2007;28:105-14.

29. Higgins JPT, Thomas J, Chandler J, et al. Cochrane Handbook for Systematic Reviews of Interventions version 6.0 (updated July 2019). Cochrane, 2019. Available online: www.training.cochrane.org/handbook

30. Furukawa TA, Barbui C, Cipriani A, et al. Imputing 
missing standard deviations in meta-analyses can provide accurate results. J Clin Epidemiol 2006;59:7-10.

31. Guyatt G, Oxman AD, Akl EA, et al. GRADE guidelines: 1 . Introduction-GRADE evidence profiles and summary of findings tables. J Clin Epidemiol 2011;64:383-94.

32. Norris, SL, Meerpohl JJ, Akl EA, et al. The skills and experience of GRADE methodologists can be assessed with a simple tool. J Clin Epidemiol 2016;79:150-8.e1.

33. Cao B, Wang Y, Wen D, et al. A Trial of LopinavirRitonavir in Adults Hospitalized with Severe Covid-19. N Engl J Med 2020. [Epub ahead of print].

34. Li Y, Xie Z, Lin W, et al. An exploratory randomized, controlled study on the efficacy and safety of lopinavir/ ritonavir or arbidol treating adult patients hospitalized with mild/moderate COVID-19 (ELACOI). 2020. [medRxiv preprint]. Available online: https://www.medrxiv. $\mathrm{org} /$ content/10.1101/2020.03.19.20038984v2

35. Chen J, Ling Y, Xi X, et al. Efficacies of lopinavir/ ritonavir and abidol in the treatment of novel coronavirus pneumonia. Chin J Infect Dis 2020;38:E008.

36. Chan KS, Lai S, Chu C, et al. Treatment of severe acute respiratory syndrome with lopinavir/ritonavir: A multicentre retrospective matched cohort study. Hong Kong Med J 2003;9:399-406.

37. Chu CM, Cheng V, Hung I, et al. Role of lopinavir/ ritonavir in the treatment of SARS: initial virological and clinical findings. Thorax 2004;59:252-6.

38. Deng L, Li C, Zeng Q, et al. Arbidol combined with $\mathrm{LPV} / \mathrm{r}$ versus LPV/r alone against Corona Virus Disease 2019: A retrospective cohort study. J Infec 2020. [Epub ahead of print]

39. Loutfy MR, Blatt LM, Siminovitch KA, et al. Interferon Alfacon-1 Plus Corticosteroids in Severe Acute Respiratory Syndrome: A Preliminary Study. JAMA 2003;290:3222-8.

40. Li J, Wu H, Huang Z, et al. Clinical study on treatment of severe acute respiratory syndrome with interferon-alpha. Clin Med Chin 2005;21:109-10.

41. Liu D, Ouyang J, Zou M, et al. Antiviral Agents in Treating Severe Acute Respiratory Syndrome: A Multifactorial Analysis. Chin J Nosocomiol 2009;19:434-6.

42. Xu M, Cai W, Jia W. Therapeutic efficacy in patients with infectious atypical pneumonia: comparison of different protocols. Chin J Pract Intern Med 2003;23:21-3.

43. Shalhoub S, Farahat F, Al-Jiffri A, et al. IFN-alpha2a or IFN-beta1a in combination with ribavirin to treat Middle East respiratory syndrome coronavirus pneumonia: a retrospective study. J Antimicrob Chemother 2015;70:2129-32.
44. Leong HN, Ang B, Earnest A, et al. Investigational use of ribavirin in the treatment of severe acute respiratory syndrome, Singapore, 2003. Trop Med Int Health 2004;9:923-7.

45. Chiou HE, Liu C, Buttrey MJ, et al. Adverse Effects of Ribavirin and Outcome in Severe Acute Respiratory Syndrome. Chest 2005;128:263-72.

46. Lau EH, Cowling BJ, Muller MP, et al. Effectiveness of ribavirin and corticosteroids for severe acute respiratory syndrome. Am J Med 2009;122:1150.e11-21.

47. Wang J, Li D, Li J, et al. Effects of anti-virus agents for the dosing period of glucorticoids in SARS patients. Chin J New Drug 2005;14:109-10.

48. Muller MP, Dresser L, Raboud J, et al. Adverse events associated with high-dose ribavirin: evidence from the Toronto outbreak of severe acute respiratory syndrome. Pharmacotherapy 2007;27:494-503.

49. Guo L, Han Y, Li J, et al. Long-term outcomes in patients with severe acute respiratory syndrome treated with oseltamivir: A 12-year longitudinal study. Int J Clin Exp Med 2019;12:12464-71.

50. Xu Y, Chen S, Jiang M. Clinical observation of oseltamivir phosphate in the treatment of severe acute respiratory syndrome. Chin J Clinical Rational Drug Use 2011;4:68-9.

51. Omrani AS, Saad MM, Baig K, et al. Ribavirin and interferon alfa-2a for severe Middle East respiratory syndrome coronavirus infection: a retrospective cohort study. Lancet Infect Dis 2014;14:1090-5.

52. Arabi YM, Shalhoub S, Mandourah Y, et al. Ribavirin and Interferon Therapy for Critically Ill Patients with Middle East Respiratory Syndrome: A Multicenter Observational Study. Clin Infect Dis 2020;70:1837-44.

53. Chen C, Huang J, Cheng Z, et al. Favipiravir versus Arbidol for COVID-19: A Randomized Clinical Trial 2020; MedRxiv 2020. [cited 2020 Apr 2]. Available online: https://www.medrxiv.org/content/10.1101/2020.03.17.200 37432v3

54. Chen J, Liu D, Liu L, et al. A pilot study of hydroxychloroquine in treatment of patients with common coronavirus disease-19 (COVID-19). J Zhejiang Univ 2020. doi: $10.3785 /$ j.issn.1008-9292.2020.03.03.

55. Chen Z, Hu J, Zhang Z, et al. Efficacy of hydroxychloroquine in patients with COVID-19: results of a randomized clinical trial. MedRxiv 2020. [cited $2020 \mathrm{Apr}$ 2]. Available online: https://www.medrxiv.org/content/10.1 101/2020.03.22.20040758v3

56. Cai Q, Yang M, Liu D, et al. TEMPORARY REMOVAL: Experimental Treatment with Favipiravir for COVID-19: 
An Open-Label Control Study. Engineering (Beijing) 2020. [Epub ahead of print].

57. Yang B, Zhu Y. Epidemiology and treatment of novel coronavirus pneumonia (COVID-19) in children and adults. Journal of Xi' an Jiaotong University (Medical Sciences) 2020.

58. She J, Liu W. Epidemiological characteristics and prevention and control measures of Corona Virus Disease 2019 in children. Journal of Tropical Medicine 2020.

59. Rothe C, Schunk M, Sothmann P, et al. Transmission of 2019-nCoV Infection from an Asymptomatic Contact in Germany. N Engl J Med 2020;382:970-1.

60. Lai CC, Shih T, Ko W, et al. Severe acute respiratory syndrome coronavirus 2 (SARS-CoV-2) and coronavirus disease-2019 (COVID-19): The epidemic and the challenges. Int J Antimicrob Agents 2020;55:105924.

61. Chen F, Liu Z, Zhang F, et al. Frist case of severe childhood novel coronavirus pneumonia in China. Zhonghua Er Ke Za Zhi 2020;58:179-82.

62. Wang D, Ju X, Xie F, et al. Clinical analysis of 31 cases of 2019 novel coronavirus infection in children from six provinces (autonomous region) of northern China. Chin J Pediatr 2020;58(4).

63. Zhenyu F, Li P, Jun L, et al. Clinical Features of COVID-19 Related Liver Damage. 2020; MedRxiv 2020. [cited 2020 Apr 2]. Available online: https://www.medrxiv. org/content/10.1101/2020.02.26.20026971v2

Cite this article as: Shi Q, Zhou Q, Wang X, Liao J, Yu Y, Wang Z, Lu S, Ma Y, Xun Y, Luo X, Li W, Fukuoka T, Ahn HS, Lee MS, Luo Z, Liu E, Chen Y, Li Q, Yang K, Guan Q; on behalf of COVID-19 Evidence and Recommendations Working Group. Potential effectiveness and safety of antiviral agents in children with coronavirus disease 2019: a rapid review and meta-analysis. Ann Transl Med 2020;8(10):624. doi: 10.21037/atm-20-3301
64. Young BE, Sean WX, Shirin K, et al. Epidemiologic Features and Clinical Course of Patients Infected With SARS-CoV-2 in Singapore. JAMA 2020;323:1488-94.

65. Gautret P, Lagier JC, Parola P, et al. Hydroxychloroquine and azithromycin as a treatment of COVID-19: results of an open-label non-randomized clinical trial. Int J Antimicrob Agents 2020. [Epub ahead of print].

66. Falzarano D, Wit E, Rasmussen AL, et al. Treatment with interferon-alpha $2 \mathrm{~b}$ and ribavirin improves outcome in MERS-CoV-infected rhesus macaques. Nat Med 2013;19:1313-7.

67. Stockman LJ, Bellamy R, Garner P. SARS: Systematic Review of Treatment Effects. PLoS Med 2006;3:e343.

68. Hart BJ, Dyall J, Postnikova E, et al. Interferon- $\beta$ and mycophenolic acid are potent inhibitors of Middle East respiratory syndrome coronavirus in cell-based assays. J Gen Virol 2014;95:571-7.

69. Holshue ML, DeBolt C, Lindquist S, et al. First case of 2019 novel coronavirus in the United States. N Engl J Med 2020. [Epub ahead of print].

70. Wang M, Cao R, Zhang L, et al. Remdesivir and chloroquine effectively inhibit the recently emerged novel coronavirus (2019-nCoV) in vitro. Cell Res 2020. [Epub ahead of print].

71. Grein J, Ohmagari N, Shin D, et al. Compassionate Use of Remdesivir for Patients with Severe Covid-19. N Engl J Med 2020. [Epub ahead of print]. 